\title{
Editorial announcements and information - ICFA Beam Dynamics Newsletter No. 81
}

\author{
Ingo Hofmann $a, b, 1$ Vladimir Shiltsev $c, 2$ \\ ${ }^{a}$ GSI Helmholtzzentrum fur Schwerionenforschung GmbH, Planckstr. 1, D-64291 Darmstadt, Germany \\ ${ }^{b}$ Technische Universität Darmstadt, Schlossgartenstr. 8, D-64289 Darmstadt, Germany \\ ${ }^{c}$ Fermi National Accelerator Laboratory, P.O. Box 500, Batavia, IL, USA 60510 \\ E-mail: i.hofmann@gsi.de; shiltsev@fnal.gov
}

\begin{abstract}
This is a general information article of the ICFA Beam Dynamics Newsletter No. 81. It contains two forewords from the Editor-in-Chief and the issue editor, a workshop and conference report section, a recent Ph.D. thesis section, a forthcoming beam dynamics events section, and a section of announcements from the beam dynamics panel.
\end{abstract}

KEYwORDS: Accelerator modelling and simulations (multi-particle dynamics, single-particle dynamics); Beam dynamics; Beam optics

1 Editor-in-Chief

2 Issue Editor 


\section{Contents}

1 From the Panel Chair (Editor-in-Chief) 2

2 From the Issue Editor 2

3 Workshop and Conference Reports 3

3.1 US Accelerator and Beam Physics Community Workshops (2019-2020) 3

3.2 Mitigation Approaches for Hadron Storage Rings and Synchrotrons (June 22-24 and June 29 - July 1, 2020)

3.3 HKUST IAS HEP Conference and Workshop on Plasma Accelerators (January 18-21, 2021) (1)

3.4 ARIES Workshop on Storage Rings and Gravitational Waves (SRGW2021, February 2 - March 11, 2021)

4 Recent Doctoral Theses

4.1 Optimizing the Radioisotope Production of the Novel AMIT Superconducting Weak Focusing Cyclotron

4.2 On Systematics and their Mitigation in MAGIS-100 Atomic Interferometer Experiment to Explore the Dark Sector and Early Universe

4.3 Nonlinear Integrable Optics Experiment and Diagnostics in IOTA

4.4 Direct Observation of the Hosing Instability of a Long Relativistic Proton Bunch in $\begin{array}{ll}\text { the AWAKE Experiment } & 10\end{array}$

4.5 Precise Simulations of Multibunches in High Intensity Cyclotrons 11

4.6 From Wave Propagation to Spin Dynamics: Mathematical and Computational Aspects 11

4.7 Compact Radio-frequency Quadrupoles for Industrial and Medical Applications 12

5 Forthcoming Beam Dynamics Events $\quad 13$

5.1 Snowmass'21: Accelerator Frontier 13

5.2 The One World charged particLe accElerator (OWLE) Colloquium \& Seminar Series 15

5.3 DPB Events at the APS April Meeting 2021 (April 17-20, 2021) 15

6 Book announcements $\quad 17$

6.1 Safety for Particle Accelerators 17

6.2 The Science and Technology of Particle Accelerators 17

6.3 Accelerator Technology: Applications in Science, Medicine, and Industry 18

6.4 Particle Physics Reference Library_Volume 3: Accelerators and Colliders 19

6.5 Obsessed by a Dream: The Physicist Rolf Wideroe-a Giant in the History of $\begin{array}{ll}\text { Accelerators } & 20\end{array}$ 


\title{
1 From the Panel Chair (Editor-in-Chief)
}

\author{
Ingo Hofmann, GSI and Technical University Darmstadt, Germany
}

This issue of the ICFA Beam Dynamics Newsletter is the third one in this series to appear online as Special Issue of the Journal of Instrumentation. Special thanks go to Vladimir Shiltsev as the issue editor for coordinating a comprehensive and impressive set of articles covering the status and progress of the electron lens developments worldwide. The presented articles mirror the major accelerator facilities and projects that employ or plan to use electron lenses for beam-beam compensation, collimation, space-charge compensation or other applications.

The Workshop and Conference Reports section this time includes several online events, which reflect the remarkable creativity of our community - all in the attempt of making the best out of the current circumstances, which have prevented person-to-person meetings.

As it is our tradition, the Recent Doctoral Theses section includes a number of short reports on relevant theses in the field of accelerators, which are brought to the attention of the editors.

The Forthcoming Beam Dynamics Events section announces two online meetings. The series of ICFA Advanced Beam Dynamics Workshops is still interrupted due to the pandemic. eeFACT2020, the 64th ICFA Advanced Beam Dynamics Workshop on High Luminosity Circular e+e- Colliders, originally scheduled for 14-16 September 2020 on Elba, Italy, is now scheduled to 11-16 September 2022; for HB2020, the 65th ICFA Advanced Beam Dynamics Workshop on High-Intensity and High-Brightness Hadron Beams, originally scheduled for 5-9 October 2020 at FNAL and rescheduled to October 2021, a final decision is still pending; and ERL21, the 66th ICFA Advanced Beam Dynamics Workshop on Energy Recovery Linacs, planned at Cornell University, is postponed to after the pandemic.

The new Book Announcements section presents books in our field that have appeared in 2020/21 - thanks to Jens Vigen (CERN) for having compiled this section.

I am glad to announce that Yunhai Cai (SLAC) has accepted to be editor of the next Newsletter No.82 - planned for August 2021. Its theme is "Advanced Accelerator Modelling", a very dynamically evolving field including topics like machine learning, advanced methods and concepts in computation and control, and others.

\section{From the Issue Editor}

Vladimir Shiltsev, Fermilab, Batavia, IL, USA

The first electron lenses - understood as "focusing elements made of electrons" rather than "lenses to focus electrons" - were proposed in the mid-1990s for compensation of beam-beam effects. Since then, these novel instruments for high-energy particle accelerators have been found particularly useful for superconducting hadron colliders. They were built and employed in the Tevatron proton-antiproton collider at Fermilab in the 2000s and in the Relativistic Heavy Ion Collider at BNL in the 2010s, and they are being designed and built nowadays for the high-luminosity upgrade of the Large Hadron Collider at CERN. The richness of the concept of employment of lowenergy high-current bright electron beams in high-energy accelerators, its technological feasibility, and the flexibility of the method have resulted in a wide range of high-impact applications and 
proposals - from compensation of long-range and head-on beam-beam effects to Landau damping, from the beam halo scraping with hollow electron beams to space-charge compensation, from selective resonant extraction to longitudinal collimation, from the use of electron beams as nonlinear integrable optics elements to a beam-beam kicker, etc. Electron lenses have shown themselves as remarkably fertile and dynamic novel accelerator tools, and they are still breaking new ground and inspire new applications. In the coming decades, one can foresee expansion of the method beyond supercolliders to other accelerators, such as, e.g., high-intensity proton machines, including space-charge dominated rapid cycling synchrotrons, e+e- colliders, etc., resulting in better, more effective, and more economical machines. In the past, short reports and discussions on the electron lens-related topics were published in the ICFA Beam Dynamics Panel Newsletters No.16 (1998), No.32 (2003), No.34 (2004), No.44 (2007), No.61 (2013) and No.75 (2018).

This Special Issue of JINST is the most comprehensive collection to date of articles presenting the progress of the electron lenses world-wide. It includes contributions from many groups and laboratories which are actively working on the development of the electron lenses and their applications. I greatly appreciate the hard work by all authors for their excellent contributions to this issue of the ICFA Beam Dynamics Newsletter. This collection includes papers on electron lenses by two dear colleagues who, alas, are no longer with us - Yuri Alexahin (1948-2020) and Vyatcheslav "Slava" Danilov (1966-2014). They both were critical to the development of the electron lens methods, especially at the critical early stages. The relevance, the beauty of the key ideas, and the subtlety of mathematical craftsmanship are just as characteristic of the articles included here as of the scientific inheritance from Yuri and Slava for each of them as a whole. We publish these papers with a feeling of gratitude to these brilliant scientists, with whom some of us had the honor of many years of fruitful collaboration and friendship.

\section{Workshop and Conference Reports}

\subsection{US Accelerator and Beam Physics Community Workshops (2019-2020)}

\section{S. Nagaitsev (FNAL)}

nsergei@fnal.gov

Accelerators are a key capability for enabling discoveries in many fields such as elementary particle physics, nuclear physics, and materials sciences. While recognizing the past dramatic successes of accelerator-based particle physics research, the April 2015 report of the Accelerator Research and Development Subpanel of HEPAP https: //www . osti . gov/biblio/1320612 recommended the development of a long-term vision and a roadmap for accelerator science and technology to enable future US DOE HEP capabilities.

An effort to gather community input to prepare for the development of the Accelerator and Beam Physics (ABP) Roadmap by the US DOE Office of Science/HEP General Accelerator R\&D (GARD) program has now been completed. Two preparatory workshops were held, an in-person workshop at LBNL in December of 2019 https://conferences.lbl.gov/event/279/ and a virtual workshop hosted by FNAL and ANL in April-May of 2020 https://indico. fnal. gov/event/22709/. These workshops convened university and laboratory scientists, active in the ABP research, to develop an $R \& D$ roadmap for the long-term vision for enabling future US 
DOE HEP accelerator capabilities. The workshops were organized around four Grand Challenges: Intensity, Beam Quality, Beam Control and Beam Prediction. Research topics with milestones were established for each of the Grand Challenges. The workshop report documents the outcome of these workshops and will be further used to establish the ABP Roadmap for the US GARD program - see https://arxiv.org/abs/2101.04107.

\subsection{Mitigation Approaches for Hadron Storage Rings and Synchrotrons (June 22-24 and} June 29 - July 1, 2020)

\section{G. Franchetti (GSI), F. Zimmermann (CERN) \\ g.franchetti@gsi.de,frank.zimmermann@cern.ch}

The ARIES-APEC workshop "Mitigation Approaches for Hadron Storage Rings and Synchrotrons" was held over six days, from 22 to 24 June and from 29 June to 1 July 2020, in a safe virtual space. The virtual format of the workshop provided the flexibility of planning the various sessions so as to ensure optimum conditions for a fruitful scientific exchange between regions and communities. This ARIES workshop addressed the mitigations of the mechanisms leading to beam degradation in hadron rings. The state of the art of relevant techniques and the present understanding of underlying dynamics were reviewed. The workshop featured 33 invited contributions and several long and animated discussions. The Scientific Program Committee consisted of Mei Bai (GSI and U. Bonn, Germany), Giuliano Franchetti (GSI and GU Frankfurt, Germany), Shinji Machida (RAL, UK), Kazuhito Ohmi (KEK, Japan), Tatiana Pieloni (EPFL, Switzerland), Qing Qin (IHEP Beiiing, China), Vladimir Shiltsev (FNAL. U.S.A.), Rogelio Tomas (CERN, Switzerland), and Frank Zimmermann (CERN, Switzerland).

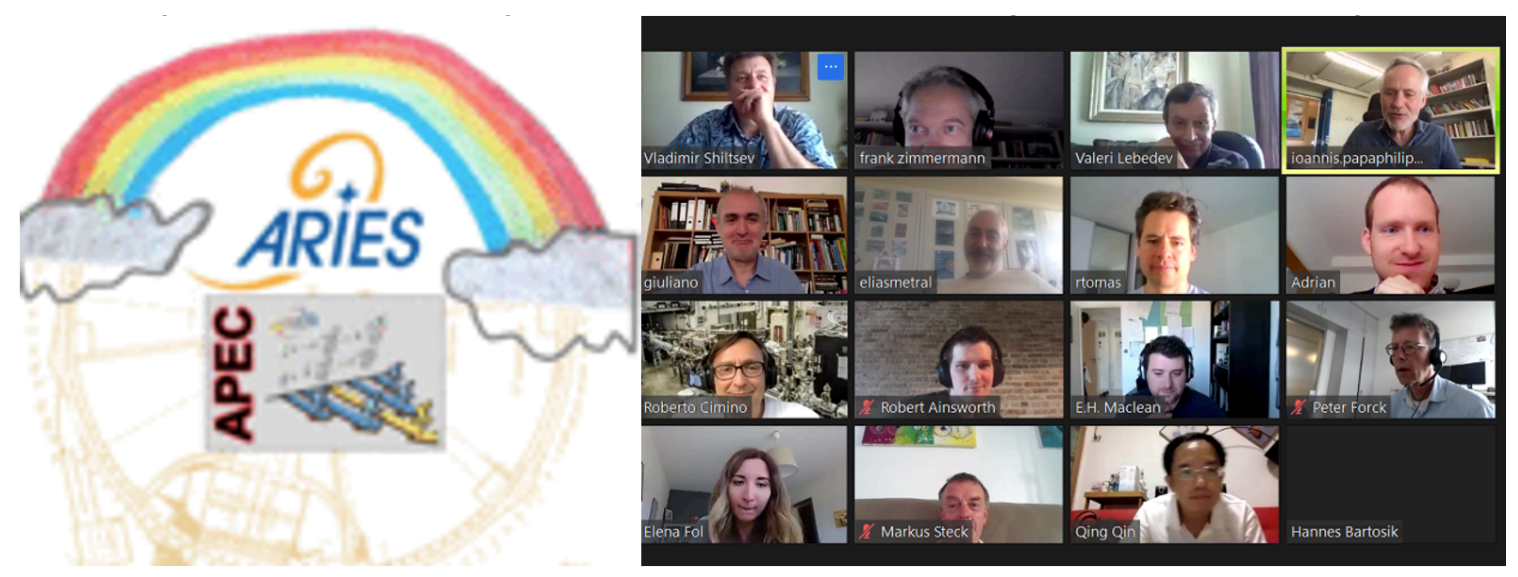

Figure 1. Workshop logo (left) and a snapshot of some of the zoom participants.

The workshop was attended by 118 participants, hailing from the following institutions: CAS IHEP Beijing, CAS IMP Lanzhou, Spallation Neutron Source Science Center, IJCLab, CERN, CEA Saclay, IRFU/DACM/LEDA, GSI, Goethe University Frankfurt, Karlsruhe Institute of Technology, TU Darmstadt, INFN - Sezione di Napoli, INFN - Laboratori Nazionali di Frascati, INFN-Naples Unit, University of Rome la Sapienza - INFN-Roma1, LNF - INFN, KEK/J-PARC, J-PARC/JAEA, KEK-ACCL/IHEP, STFC/RAL/ISIS, University of Oxford, Fermilab, Brookhaven National Lab, 
ORNL, Fermilab/UChicago, Indiana University, SLAC National Accelerator Laboratory, TU Vienna / CERN, and Ecole Polytechnique Federale de Lausanne (EPFL). 23 of the participants were women (19.5\%). The distribution by country was as follows - Austria: 1 (TU Vienna), China: 7 (IHEP, IMP, CSNS), France: 5 (IJCLab, CEA Sacaly), Germany: 18 (Goethe U. , GSI, KIT), Italy: 5 (INFN-LNF, INFN-Napoli, U. La Sapienza), Japan: 10 (KEK, JPARC/JAEA), Russia: 5 (BINP, JINR), Switzerland: 37 (CERN, EPFL), UK: 12 (STCF/RAL/ISIS, U. Oxford), USA: 18 (BNL, FNAL, ORNL, U. Indiana, SLAC).

The workshop was composed of the following sessions/blocks: 1. Optics and nonlinear dynamics; 2. High intensity - instabilities; 3. High intensity - electron cloud and vacuum; 4. Laboratory experience; 5 . Beam loss control; and 6. Special measures.

The workshop unveiled numerous intriguing points and questions, for example:

1 extending Yong Ho Chin's 1985 Landau-damping approach with 2D detuning and feedback; Sacherer and Hofmann-Pedersen models surpassed by Andrei Lebedev and G van Kampen; MELODY solves Lebedev equation; noise induced diffusion modifies stability diagram; sequential $\mathrm{H}$ - resonance excitation by laser opens path towards arbitrary painting without foil constraints;

2 the mystery of LHC's shifted IP waists, chromatic beta beating at the LHC collision point; are we meeting the beam-beam tolerance?; quest for a better fringe-field model; Autoencoder Neural Networks to denoise and simulate missing data; Should all future machines go nonlinear?; beta functions with space charge or beam-beam;

3 can the next-generation machines be made electron-cloud free? laser treatment and/or a-C coating as options for future unclouded machines; how could the CERN complex go beyond the LIU beam brightness?; FAIR vacuum chambers serve as pump; space-charge mitigations: resonance compensation and changing the integer tune to avoid structural resonances;

4 which effects or constraints are limiting the performance of ISIS, RHIC/EIC, and FNAL complex? ; what are the present or expected space-charge limits? J-PARC RCS focus on loss reduction, painting, 2nd harmonic RF, compensating two 3rd order resonances; CSNS horizontal instability driven by resistive wall mitigated by tune variation pattern and control; GSI tune wobble to improve spill quality; EIC option to replace hadron beam every hour; no impact of cooling on polarization expected; FNAL path to 1.2 MW on LBNF target based on new $800 \mathrm{MeV}$ SC linac, increased booster rate, and shorter MI cycle; losses in booster limit FNAL accelerator complex performance and mitigation through higher injection energy, painting, and two-stage collimation; $1 \%$ beta beating in LHC, a few percent in CERN SPS; either modelling should become more realistic, or hardware should more closely approach the ideal requirement;

5 LHC beam 1 horizontally affected by high-frequency cluster noise, around $8 \mathrm{kHz}$; GALACTIC Vlasov Solver (water bag) and BimBim code (Gaussian) indicate Space Charge reduces TMCI threshold by factor 10-20; SIS100 lattice optimized for maximum catching efficiency; active SC compensation will be benchmarked at IOTA; hollow electron lens and crystal collimators part of HL-LHC baseline; future option: magnetized or electrostatic collimators; 
6 synchrotron oscillations improve slow extraction quality; different cooling schemes are used for injection and for the stacked orbit; optical stochastic cooling experiment in IOTA and proposal for ring-based electron cooling,

The virtual Mitigations workshop proved a great success. Discussions continued well outside the regular workshop hours, arguably physics more intensely than during evening discussions at live workshops. It was so popular that, aside from the registered 118 participants, several unknown attendees were equally spotted, going by names such as "Honor", "Huawei Mate 20", "LiZip", etc. The Mitigations 2020 workshop was managed and supported by six scientific secretaries: Alexander Engeda (U. Frankfurt), Elena Fol (U. Frankfurt and CERN), Michael Hofer (TU Wien and CERN), Annemarie Lauterbach (U. Frankfurt), Giulia Russo (U. Frankfurt and CERN), Tirsi Prebibaj (U. Frankfurt and CERN). A comprehensive summary report composed, compiled and edited by the scientific secretaries will be deposited on Zenodo. The workshop also initiated a survey on spacecharge mitigation, whose results were reported in an ARIES milestone report (ARIES M31). The details of the Mitigations workshop program as well as a collection of all talks are available on the Indico web site: https://indico.gsi.de/event/10458/.

\subsection{HKUST IAS HEP Conference and Workshop on Plasma Accelerators (January 18-21, 2021)}

Jie Gao, Institute of High Energy Physics, CAS, China gaoj@ihep.ac.cn

After the discovery of the Higgs boson, the main objectives of high-energy physics are the precise measurement of Higgs properties and searches for new physics. These objectives strongly motivate the construction of next-generation experimental facilities such as e+e- Higgs factory and $100 \mathrm{TeV}$ pp collider or muon collider. With this background, the High Energy Physics (HEP) program in IAS of Hong Kong University of Science and Technology (HKUST) had started in 2015. As part of this HEP program, a four-day conference was held during January 18-21, 2021. Before that, three two-day mini-workshops focusing on theory, experiment/detector and accelerator physics, respectively, were organized on January 14-15, 2021. Due to the COVID-19 situation, both the mini-workshops and the conference were virtual - see at https://indico.cern.ch/event/ 971970/overview.

The aim of the workshop on plasma accelerators was to give a comprehensive picture of the development status and perspective of this field towards the future, such as HEP applications, and to promote international collaborations. There were in total 18 talks, plus the workshop summary presentation, with speakers coming from Asia, Europe and America.

As for the HEP conference, it had four plenary sessions. In the accelerators part, talks covered the HL-LHC, LHeC, CEPC, FCC, BINP Super Charm Tau Factory, Super KEK B, NICA of JINR, EIC of USA, ILC, CLIC, Snowmass Accelerator Frontier Planning Status, etc. In the accelerator parallel sessions, most of the 18 talks were mainly focused on CEPC SppC and FCC. At the end of the conference, there was an engaging round table discussion with the panelists, including Hitoshi Yamomoto (Tohoku University), Michael Benedikt (CERN), Raman Sundrum (University of Maryland), Steinar Stapnes (CERN), Tao Han (University of Pittsburgh), Vladimir Shiltsev (Fermilab), and Yifang Wang (IHEP). The panel discussions covered two topics: (1) 
Multiple roadmaps (new collider proposals) have been drawn for HEP of the next decades. It will be important to generate a world-wide strategy to synergize the ongoing and future endeavors, strengthen mutual coordination and collaborations, and eventually benefit all the parties; (2) Great progress has been made in astrophysical/cosmological/GWs observations and detections over the last decade. The achievements made or expected to be made in the near future in that area may have important impacts for HEP development in terms of scientific goals, strategies, and plans.

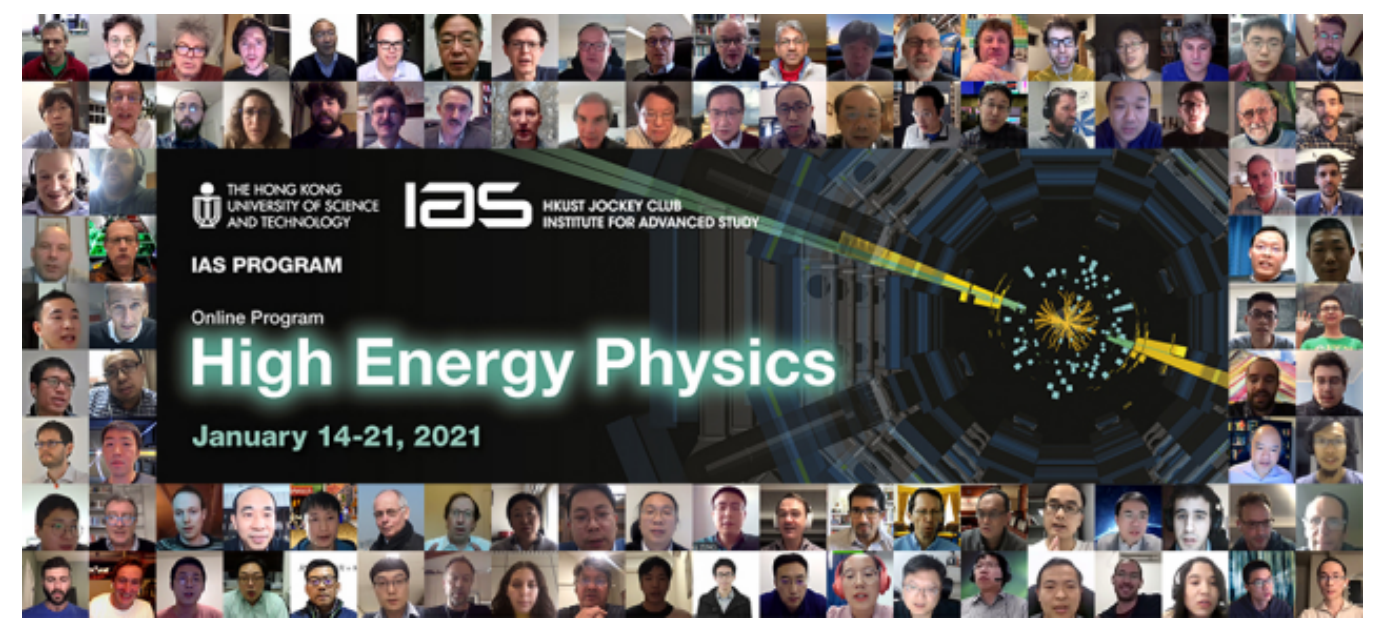

Figure 2. The 2021 HKUST IAS HEP Conference group photo.

\subsection{ARIES Workshop on Storage Rings and Gravitational Waves (SRGW2021, February 2} - March 11, 2021)

G.Franchetti (GSI Darmstadt and GU Frankfurt, Germany), M. Zanetti (University and INFN Padova, Italy), F. Zimmermann (CERN, Geneva, Switzerland)

g.franchetti@gsi.de,marco.zanetti@unipd.it,frank.zimmermann@cern.ch

Could charged-particle storage rings be used for detecting or generating gravitational waves? Proposals in this direction have appeared intermittently over the past 50 years, e.g. during and after the construction of LEP, and also for the LHC. Now that the existence of the gravitational waves has been established by LIGO and Virgo detectors, and with new larger storage rings like FCC or CEPC being put forward, this question acquires increased relevance. A topical virtual workshop organized in the frame of the EU's H2020 ARIES Work Package 6 "Accelerator Performance and Concepts" is presently shedding some light on this tantalizing possibility. About 100 storage-ring experts, accelerator scientists and members of the gravitational physics community are participating in this event for a fruitful exchange of ideas, and to jointly explore a few intriguing proposals that could pave the way for a novel direction of research. The virtual SRGW2021 workshop is organized in the format of four 2-3 hour sessions. The first session, on 2 February, was sovereignly guided by Pisin Chen of Taiwan National University. Two inspiring talks on the history of gravitational waves, by Jorge Cervantes Cota from ININ, and on the various sources of gravitational waves and their characteristics, by Bangalore Sathyaprakash from Pennsylvania State and Cardiff University, set the stage for the following developments. In the first accelerator talk of the workshop, Katsunobu 
Oide from KEK and CERN discussed various resonances that a gravitational wave could excite on a beam circulating in storage rings, and how the resonance strength could be enhanced by special beam-optical insertions serving as "gravitational-wave antennas". Subsequent lively discussions focused on the validity of representing the gravitational wave as a force on the beam. The second workshop session on 18 February, chaired by SY Lee from Indiana University, will review the functioning of VIRGO and LIGO, the sensitivity of LEP and LHC to effects such as the tides or earthquakes on the other side of the planet, and the ground motion situation at a new light source in China. The third sessions on 4 March, chaired by Jörg Wenninger of CERN, will review a recent proposal to measure gravitational waves at the LHC, the sensitivity of storage rings like SPring- 8 to the relic microwave background, and a theoretical review of the effect of a gravitational wave on the beam in a storage ring, which may also address the question of how the ring sensitivity depends on particle energy, species, and ring size. The final session on 11 March, whose chair will be Raffaele Flaminio from CNRS LAPP Annecy, aims at examining other uses of accelerator technologies, such a radiofrequency cavities and atomic beams, for the detection of gravitational waves, and, conversely, the expected generation of gravitational waves by a storage ring. More information on SRGW2021 is posted at https://indico.cern.ch/event/982987.

\section{Recent Doctoral Theses}

\subsection{Optimizing the Radioisotope Production of the Novel AMIT Superconducting Weak Focusing Cyclotron}

Pedro Calvo Portela, Department of Technology, CIEMAT, Madrid, Spain

Graduation date: September 2020

Institution: Universidad Complutense de Madrid

Supervisors: Dra. Concepcion Oliver Amoros

Nuclear imaging techniques are becoming one of the most widely used medical diagnostics tools for certain illness such as cancer and Alzheimer disease. The increase in these medical procedures, particularly positron emission tomography, is leading to a saturation of the actual radioisotope production system. Therefore, particle accelerators, especially the cyclotron, emerged as an alternative to the traditional supply system based on centralized production in nuclear reactors. Its characteristics from a physical and technological point of view allow a controlled and localized production, especially relevant in the case of short-lived radionuclides, through a well-known technology developed for decades without the use of large and expensive facilities. With that in mind, the AMIT project (Advanced Molecular Imaging Technologies) aims to extend the use of these medical procedures with the development of a new compact cyclotron focused on the on-site shortlife radioisotopes production, specifically ${ }^{11} C$ and ${ }^{18} F$, in hospitals and research centers. In order to achieve this main objective, the AMIT cyclotron is based on a classical weak focus configuration with high magnetic field provided by a superconducting magnet with an autonomous cryogenic system. In addition, with the aim of reducing the total size of the accelerator, the cyclotron employs an internal $\mathrm{H}^{-}$ion source with an electron stripping system that provides a final proton beam that is transported to the production target. 
This thesis evaluates the challenging combination of all the technical characteristics of the AMIT cyclotron, which results in a balance of the beam dynamics with all the subsystems to achieve an optimal radioisotope production. For this goal, all the physical processes associated with the beam acceleration, from the ions production and the injection into the accelerator to the extraction of the resulting beam and its transport to the target, are studied by means of theoretical analysis, computational calculations and experimental measurements. Firstly, the experimental results obtained in the characterization campaigns of the cyclotron ion source are presented. The output beam current, and, therefore, the fulfillment of the main requirements of the accelerator, depends largely on the current injected into the accelerator. For this reason, the knowledge related to the operation of the ion source and the resultant beam has been analyzed in a dedicated facility at CIEMAT (Centro de Investigaciones Energéticas, Medioambientales y Tecnológicas). The beam dynamics have been assessed by means of simulations carried out with the OPAL code (Object Oriented Parallel Accelerator Library). The study includes all the relevant effects from the point of view of particle accelerator physics, with special attention to those characteristics that allow the precise adjustment of the cyclotron operation and the optimization of the radioisotope production under different conditions. For this purpose, the adjustment of the different subsystems within the design tolerances and the possible modification of some of them in their final assembly are analyzed, as well as the impact of some significant parameters variations in the magnetic field, the radiofrequency system, the acceleration gap or the beam extraction system. In addition, a relevant feature in a compact $H^{-}$cyclotron with internal ion source is the beam interactions with the residual gas and the electromagnetic field. Therefore, the computational implementation of these physical processes within the OPAL code is presented, as well as their application in the AMIT cyclotron in order to minimize the beam losses and optimize the final beam current with the control of the vacuum conditions in the accelerator. All these studies allow the combination of the different elements of the cyclotron with the beam dynamics for an optimal and efficient radioisotope production, essential for the commissioning and the optimization of the cyclotron operation.

\subsection{On Systematics and their Mitigation in MAGIS-100 Atomic Interferometer Experiment to Explore the Dark Sector and Early Universe}

Jeremiah Thomas Mitchell, Department of Physics, Northern Illinois University, DeKalb, IL, USA

Graduation date: September 29, 2020

Institution: Northern Illinois University, DeKalb, Illinois, USA

Supervisors: Prof. Swapan Chattopadhyay

The thesis describes a detailed enumeration, estimation, and mitigation strategies of all possible Systematic Errors potentially affecting experimental observational data in a long baseline (100 meter) atomic interferometer set up to study the dark sector and early universe, namely the MAGIS1000 experiment at Fermilab. Such systematics include seismic noise, gravity gradient noise, residual magnetic field, pressure and temperature fluctuation, issues of a long 100-meter vertical vacuum column, Coriolis effects due to earth's rotation and various laser wavefront aberrations. A detained systematic error budget is established for the MAGIS-100 experiment and mitigation strategies outlined. The findings can be extrapolated eventually to a longer 1000-meter baseline experiment in the future, possibly in the Sanford Lab underground cave. 


\subsection{Nonlinear Integrable Optics Experiment and Diagnostics in IOTA}

Sebastian Szustkowski, Department of Physics, Northern Illinois University, DeKalb, Illinois, USA

Graduation date: October 21, 2020

Institution: Northern Illinois University, DeKalb, Illinois, USA

Supervisors: Prof. Swapan Chattopadhyay

The thesis describes a series of experiments using electron beams in the nonlinear lattice of the IOTA test accelerator at Fermilab, which has a large betatron frequency spread, aimed at exploration of mitigation of coherent instabilities and particle loss via careful balancing of lattice nonlinearities against Coulomb self-fields (i.e. space charge forces). The results are directly relevant to high intensity proton beams. A second major aspect of the thesis is to develop a minimally invasive beam instrumentation, a specially designed, fabricated, and tested Gas Jet Monitor in particular, that can detect instabilities and particle loss and thus help mitigate them via compensation schemes.

\subsection{Direct Observation of the Hosing Instability of a Long Relativistic Proton Bunch in the AWAKE Experiment}

Mathias Julius Hüther, Max Planck Institute for Physics, Technical University Munich, Germany

Graduation date: October 27, 2020

Institution: Technical University Munich, Germany

Supervisors: Prof. A. Caldwell

In this thesis we report on the first direct experimental observation of the non-axisymmetric Hosing Instability of a long relativistic proton bunch propagating through a meter-scale plasma with density $n_{p e} \sim 0.5 \cdot 10^{14} \mathrm{~cm}^{-3}$ in the AWAKE experiment at CERN. Analysing streak camera images, we observe a periodic oscillation of the proton bunch centroid with growing amplitude along the bunch. We calculate by means of a Fast Fourier Transform that the frequency of the oscillation is equal to the cold electron plasma frequency. Moreover, by capturing time-integrated radial proton bunch profiles, we show that the Hosing Instability develops along a characteristic plane in the bunch. We discuss the results of three-dimensional particle-in-cell simulations of the Hosing Instability performed in the OSIRIS code at similar parameters compared to our experiment in different propagation distances along the plasma and in different planes across the bunch. By performing fits to the bunch centroid position in simulation and experiment, we show good agreement to the theoretical model of the Hosing Instability and give evidence for Coupled Beam Hosing, i.e. the coupling of the bunch centroid oscillation to the modulation of the bunch density due to the process of Seeded Self-Modulation via the plasma wakefields. Furthermore, we show that in the case that the Self-Modulation process is not seeded, the occurrence of the Hosing Instability depends on the delay between the high-power laser pulse ionizing the Rubidium vapour and the arrival of the proton bunch. In this configuration, both events where the Self-Modulation Instability or the Hosing Instability (with and without coupling to the Self-Modulation process) develops can be observed. Eventually, we infer that the Hosing Instability can also be induced by a deliberate misalignment of the plasma channel with respect to the proton bunch trajectory. 


\subsection{Precise Simulations of Multibunches in High Intensity Cyclotrons}

Matthias Frey, PSI, Forschungsstrasse 111, 5232 Villigen PSI, Switzerland

Graduation date: 28 October 2020

Institution: ETH Zurich

Supervisors: Prof. Dr. K. S. Kirch, Dr. A. Adelmann

A low intensity loss is a key factor in the successful operation of high intensity isochronous cyclotrons. Since lost particles activate and even damage the machine and its surrounding, the operational goal is to minimize particle loss, i.e. the occurrence of halo particles. The causes of halo particles are the bunch internal space charge as well as radially adjacent bunches at low turn separation. In order to study the effect of neighboring bunches in the PSI ring cyclotron more precisely, we first developed a new trim coil model based on real profile measurements to match the turn pattern between simulation and measurement up to a mean absolute error of 1.4 mm over all turns. In a second step, the existing electrostatic particle-in-cell beam dynamics model in OPAL (Object-oriented Parallel Accelerator Library) was enhanced with block-structured adaptive mesh refinement capabilities to improve the spatial mesh resolution at reduced costs compared to regular single level particle-in-cell with uniform Cartesian grid. Furthermore, a global sensitivity study based on polynomial chaos expansion and Sobol' indices helped to identify irrelevant simulation parameters to reduce the time to solution. Finally, the effect of neighboring bunches close to extraction was analysed, showing a convergent behavior with respect to the number of bunches.

\subsection{From Wave Propagation to Spin Dynamics: Mathematical and Computational Aspects}

Oleksii Beznosov, Department of Mathematics and Statistics, University of New Mexico

Graduation date: December 2020

Institution: University of New Mexico

Supervisors: Klaus Heinemann, Desmond P. Barber, James A. Ellison

The accelerator community is looking forward to good electron spin polarization at the EIC electron ring at Brookhaven National Laboratory, and at the proposed FCC-ee at CERN and the CEPC in China. With these in mind, this dissertation takes a new look at ways of considering and calculating electron polarization. As indicated in the title, the dissertation deals, in fact, with two topics, namely wave propagation and spin dynamics. However, although these topics are apparently disparate, the skills and numerical techniques involved in dealing with wave propagation have been key to handling the numerics of the spin dynamics. It is the spin dynamics that is of interest to the accelerator community and this brief description will focus on that part of the thesis. The part of the dissertation dealing with spin presents analytical and numerical work on the so-called Bloch equation, which evolves the polarization density of an electron bunch. It is a system of three coupled equations of Fokker-Planck type, introduced in 1975 by Derbenev and Kondratenko.

The numerical work consists of directly integrating the Bloch equation for simple models and, more generally, integrating approximations to the Bloch equation, namely so-called effective Bloch equations, obtained via the Method of Averaging. The integrations are made via spectral phasespace discretization together with an implicit-explicit time discretization. These numerical methods are calibrated and validated by applying them to a model of a single spin resonance and exploiting 
a generalization of the so-called Derbenev-Kondratenko formulas. The Bloch equation is based on the system of stochastic ODEs which have served since the 1980s as a basis of Monte-Carlo spintracking codes like SITROS, Zgoubi, SLICKTRACK, PTC/FPP and Bmad, but the analytical work in the thesis uses the Bloch equation to shed new light on the Derbenev- Kondratenko formulas. The latter have been the standard analytical tools for computing the equilibrium polarization and the depolarization time. As might be expected, the Bloch equation leads to a satisfactory derivation of the Derbenev-Kondratenko formula for the depolarization time. However, it also leads to the generalization of this formula via a new recursion scheme. This recursion is based on Galerkin's method to solve the effective Bloch equation with the help of the so-called invariant spin field. By this recursion, the depolarization time from the Derbenev-Kondratenko formulas is just the leading part of an infinite recursion which hopefully establishes or abandons the reality of the mysterious correction terms to the Derbenev-Kondratenko formulas, due to so-called resonance crossing.

The thesis can be found at https://www.desy.de/ mpybar/theses.html where an animation of the evolution of a polarization density can also be found. This description has focused on the spin dynamics. Nevertheless, accelerator physicists might be interested in the numerical techniques described in the section on wave propagation.

\subsection{Compact Radio-frequency Quadrupoles for Industrial and Medical Applications}

Hermann W. Pommerenke, Faculty of Computer Science and Electrical Engineering, University of Rostock and CERN

Graduation date: 28 January 2021

Institution: University of Rostock

Supervisors: Prof. Dr. Ursula van Rienen (University of Rostock), Dr. Alexej Grudiev (CERN)

The radio-frequency quadrupole (RFQ) is a crucial component of modern linear hadron accelerators. RFQs accelerate proton or ion beams at low energies and have a major impact on the overall beam quality of the accelerator. High frequencies allow for compact and economical RFQ designs that open up many possibilities of application in industry and medicine. The present thesis is motivated by the development of two compact RFQs operating at $750 \mathrm{MHz}$.

Consequently, the thesis is organized into two parts: The first part lays out radio-frequency (RF) design, measurements, and tuning of the PIXE-RFQ. The PIXE-RFQ is a one-meter long standalone accelerator providing $2 \mathrm{MeV}$ protons for non-destructive proton-induced X-ray emission (PIXE) analysis of cultural heritage artwork. Numerical simulation and analytical techniques were used to determine optimum geometric and RF parameters of cavity, tuners, vacuum pumping ports, input power coupler, and diagnostic antennas. Thermo-mechanical and beam dynamics studies are presented to validate the design. Subsequently, RF and bead-pull measurements conducted on the PIXE-RFQ and its components are reported. The RFQ was tuned by means of movable slugs and an improved tuning algorithm, which allows for correcting both field and frequency at the same time. Very good agreement between measurements and design values was observed with regards to frequency, quality factors, and field profile. The second part of the thesis reports the development of the Carbon-RFQ, which represents a key component of a new linear accelerator for light-ion cancer therapy, accelerating carbon ions to $5 \mathrm{MeV} / \mathrm{u}$. The Carbon-RFQ features trapezoidal vanes. A new semi-analytic approach based on multipole expansion is used to describe the field of the 
trapezoidal vanes. The RFQ was split into two decoupled RF cavities, whose dipole modes were detuned by means of a novel technique based on length adjustment. The splitting is described both from the RF and the beam dynamics point of view. The Carbon-RFQ study concludes with the RF design of the full structure, including computations of the maximum surface electric field and thermal-mechanical behavior.

\section{Forthcoming Beam Dynamics Events}

\subsection{Snowmass'21: Accelerator Frontier}

\section{S.Gourlay (LBNL), T.Raubenheimer (SLAC), V.Shiltsev (FNAL)}

The Particle Physics Community Planning Exercise (a.k.a. "Snowmass'21") is organized by the Divisions of Particles and Fields (DPF), Beam Physics (DPB), Nuclear Physics (DNP), Astrophysics (DAP) and Gravitation (DGRAV) of the American Physical Society. The Snowmass' 21 is a scientific study to define the most important questions for the field and to identify promising opportunities to address them, see https://snowmass 21 .org/. It provides an opportunity for the entire particle physics community to come together to identify and document a scientific vision for the future of particle physics in the U.S. and its international partners. The P5, Particle Physics Project Prioritization Panel, will take the scientific input from the Snowmass'21 and develop a strategic plan for U.S. particle physics that can be executed over a 10 year timescale, in the context of a 20-year global vision for the field.

Snowmass'21 activities are managed along the lines of ten "Frontiers": Energy Frontier, Neutrino Physics Frontier, Rare Processes and Precision, Cosmic Frontier, Theory Frontier, Accelerator Frontier, Instrumentation Frontier, Computational Frontier, Underground Facilities, Community Engagement Frontier. The Frontiers are led by Frontier Conveners who have been nominated by the community and selected by the Snowmass'21 Steering Committee. The Frontier conveners have invited topical group conveners, drawing heavily from the original list of community-proposed nominations. This process was developed in order to provide a diverse and representative leadership including junior and senior researchers, theorists and experimentalists, and balance regarding gender, geographical distribution, and background. Snowmass' 21 community-wide meetings and workshops include Snowmass Kick-off Town-Hall meeting (virtual, April 18, 2020), Snowmass Community Planning Meeting (virtual, October 5-8, 2020), and Snowmass Summer Study (July 2022 at UW Seattle).

The Accelerator Frontier (AF) conveners are Stephen Gourlay (LBNL), Tor Raubenheimer (SLAC), and Vladimir Shiltsev (FNAL). The AF activities include discussions on high-energy hadron and lepton colliders, high-intensity beams for neutrino research, beams for "Physics Beyond Colliders", accelerator technologies, science, education and outreach, as well as the progress of core accelerator technology, including RF, magnets, targets and sources. The AF participants have submitted Lettersof-Interest, contributed papers, are taking part in corresponding workshops and events, and will contribute to writing summaries and take part in the general Snowmass' 21 events.

There are 7 AF topical groups led by internationally recognized researchers: AF1 "Beam Physics and Accelerator Education" - Mei Bai (GSI), Zhirong Huang (SLAC), Steve Lund (MSU); AF2 "Accelerators for Neutrinos" - John Galambos (ORNL), Bob Zwaska (FNAL), Gianluigi Arduini 
(CERN); AF3 "Accelerators for EW/Higgs" - Georg Hoffstaetter (Cornell), Qing Qin (ESRF), Marc Ross (SLAC); AF4 "Multi-TeV Colliders" - Mark Palmer (BNL), Nadia Pastrone (INFN), Jingyu Tang (IHEP), Alexander Valishev (FNAL); AF5 "Accelerators for Physics Beyond Colliders and Rare Processes" - Mike Lamont (CERN), Richard Milner (MIT), Eric Prebys (UC Davis); AF6 "Advanced Accelerator Concepts" - Ralph Assmann (DESY), Cameron Geddes (LBNL), Mark Hogan (SLAC), Pietro Musumeci (UCLA); AF7 "Accelerator Technology - RF" Emilio Nanni (SLAC), Sergey Belomestnykh (FNAL), Hans Weise (DESY); "Magnets" - Susana Bermudez (CERN), Gianluca Sabbi (LBNL), Sasha Zlobin (FNAL); "Targets/Sources" - Charlotte Barbier (ORNL), Frederique Pellemoine (FNAL), Yin-E Sun (ANL).

The key AF questions include: What is needed to advance the physics? What is currently available (state of the art) around the world? What new accelerator facilities could be available in the next decade (or next next decade)? What $R \& D$ would enable these future opportunities? What are the time and cost scales of the $R \& D$ and associated test facilities, as well as the time and cost scale of the facilities?

By the time of the Snowmass Community Planning Meeting, 329 Letters-of-Interest (LoI) had been submitted to all seven topical groups of the Accelerator Frontier. For example, 61 LoIs submitted to AF1 (Beam Physics) covered several main themes and directions of the study, including physics of Multi-TeV colliders and ultimate beams (intensity, energy, brightness); fundamental beam physics (space-charge, plasma, beam cooling, electron lenses, ERL, instabilities, etc); modeling, AI and machine learning; education, outreach, diversity - centers/programs for general research/training; and sustainability and energy management of accelerators.

As part of the Snowmass'21 community discussion, AF1 (Accelerator Science, Education, Outreach), AF4 (Multi-TeV Colliders) and AF6 (Advanced Accelerator Concepts) have launched a series of joint workshops on the topic of Physics Limits of Ultimate Beams. The main scope of this series is to engage the community to explore fundamental ultimate beams for various physics goals, to understand the required luminosity scaling with energy and to evaluate the potential and feasibility of advanced concepts towards the ultimate physics limits, such as PeV beams yet low luminosity etc. See past and planned workshops at https://indico . fnal . gov/event/46645/, https://indico.fnal.gov/event/46742/,

https://indico.fnal.gov/event/47217/,

https://indico. fnal.gov/event/47819/.

Status and plans of other AF topical group activities can be found at https://snowmass21.org/ accelerator/.

One of the key questions for the Snowmass'21 Accelerator Frontier is “... What are the time and cost scales of the R\&D and associated test facilities as well as the time and cost scale of the facility?". It was quickly realized that there is a very large number of accelerator projects under active discussion by the HEP community: ILC, Muon Collider, gamma-gamma and ERL options, large circumference electron rings, and large circumference hadron rings, amongst others.

The AF Implementation Task Force (ITF) has been organized and charged with developing metrics and processes to facilitate a comparison between projects. The ITF is chaired by Thomas Roser (BNL) and comprises 9 world-renowned accelerator experts from Asia, Europe and the US and two representatives of the Snowmass Young (the Snowmass'21 organization of early career researchers). Because of the COVID-19 pandemic, the Snowmass'21 Report and the Community Summer Study 
meeting (CSS) - originally planned for the Summer of 2021 - will be delayed by one year until July 2022. Correspondingly shifted are the timelines: the White Paper submissions will take place by March 15, 2022; preliminary reports by the Topical Groups due May 31, 2022; preliminary reports by the Frontiers due June 30, 2022; all final reports by Topical Groups and Frontiers due September 30, 2022; the Snowmass'21 Book and the on-line archive documents due October 31, 2022.

\subsection{The One World charged particLe accElerator (OWLE) Colloquium \& Seminar Series}

\section{A. Adelmann (PSI) for the OWLE organizing committee andreas.adelmann@psi.ch}

Given the impossibility of travel during the COVID-19 crisis, the One World charged particLe accElerator (OWLE) Colloquium \& Seminar Series was established as an inter-institutional and global online event. Since September 2020, more than 15 seminars and colloquia took place with an average number of attendees of about 50. This on-line event features at the moment two scientific tracks:

- the OWLE-Colloquium is aimed at giving researchers a platform to share research and development results of very broad interest. Colloquium talks are held via Zoom once a month on the first Tuesday at 1:30 PM EST (19:30 CET, 10:30 AM PST),

- the OWLE-ML seminar series has a topical focus on machine learning and experimental demonstration of AI-ML. Seminars are held every second and last Tuesdays at 2:30 PM EST (20:30 CET, 11:30 AM PST).

The OWLE homepage is at: https://sites.google.com/view/owle/home. Here you will find past talks and the announcement of future events. Please subscribe to the OWLE YouTube channel https://www . youtube.com/channel/UCfF $48 \mathrm{fk} 7 \mathrm{VBuzCinIe}$ _-j $_{\text {sRQ }}$ and receive the latest recordings. In order to receive email announcements about upcoming talks, send email to owle@lists.psi.ch with subject "subscribe owle YOURNAME".

On the OWLE homepage you will find the list of co-organizers. Feel free to contact any of them, if you want to give a colloquium or talk at the ML seminar series.

\subsection{DPB Events at the APS April Meeting 2021 (April 17-20, 2021)}

S. Cousineau( DPB Chair, DPB APR21 Program Co-Chair, ORNL, Oak Ridge, U.S.A.), N. Pastrone(Coordinator of APR21 Muon-Collider Mini-Symposia, INFN, Torino, Italy), F. Zimmermann (DPB Chair-Elect, DPB APR21 Program Chair, CERN, Geneva, Switzerland)

csousine@ornl.gov,nadia.pastrone@to.infn.it,frank.zimmermann@cern.ch

With the invaluable help of the past and present Members-at-Large of the APS Division of Physics of Beam's (DPB's) Executive Committee (Mei Bai, John Byrd, Georg Hoffstaetter, Eric Prebys, Todd Satogata, Evgenya Smirnova, Mike Syphers) and with input from other incoming and outgoing members of the DPB Chair line (Camille Ginsburg, Michiko Minty, Sergei Nagaitsev, Vladimir Shiltsev), an attractive set of five invited and seven contributed DPB sessions have been set up for the APS April Meeting 2021 (APR21). 
Table 1. DPB program for the APS April Meeting 2021

\begin{tabular}{lll}
\hline Day, Time & Theme & Chair \\
\hline Saturday, 17 April & & \\
10:45 am - 12:33 pm & Muon Collider Symposium I & Nadia Pastrone \\
1:30 pm - 3:18 pm & Muon Collider Symposium II & Vladimir Shiltsev \\
3:45 pm - 5:33 pm & Lepton Machines and Machine Learning & Georg Hoffstaetter \\
Sunday 18 April & & \\
8:30 am - 10:18 am & Accelerators for Society (invited) & Sarah Cousineau \\
10:45 am - 12:33 pm & Muon Collider Symposium III & Eric Prebys \\
1:30 pm - 3:18 pm & In Memory of Alvin Tollestrup (inv., with DPF) & Young-Kee Kim \\
3:45 pm - 5:33 pm & Wilson Prize Award Ceremony (inv., with DPF) & Joel Butler \\
Monday 19 April & & \\
1:30 pm - 3:18 pm & Transformative Technologies (invited) & Frank Zimmermann \\
3:45 pm - 5:21pm & Advanced Acceleration, Lasers, \& Diagnostics & Mei Bai \\
Tuesday 20 April & & \\
10:45 am - 12:33 pm & Electron-Ion Collider (invited, joint with DNP) & Kent Paschke \\
1:30 pm - 3:18 pm & Muon Collider Symposium IV & Lian Tao Wang \\
3:45 pm - 5:33 pm & Hadron-Lepton Machines, $h$ - and $v$-Beams & Todd Satogata \\
\hline
\end{tabular}

Two invited pure DPB sessions address Accelerators for Society (COVID-19 research at photon and neutron sources, and the production of medical isotopes) and Transformative Technologies (today's X-FEL landscape, machine learning in accelerator science, and lasers for plasma wakefield acceleration). A joint session with the DNP presents the accelerator design of the Electron Ion Collider. One joint session with the DPF is devoted to the memory of Alvin Tollestrup (1924-2020). Another common event with the DPF is the Wilson Prize session, which includes the deliveries of the DPB doctoral thesis award (this year's winner: Lieselotte Obst-Huebl) and of two DPB special service awards (for Ernest Malamud and Stephen Holmes).

The invited sessions are complemented by three contributed DPB sessions, with nine talks each, addressing the broad themes Lepton Machines and Machine Learning; Advanced Acceleration, Lasers and Diagnostics; and Hadron-Lepton Machines, Hadron and Neutrino Beams.

Another spectacular and novel highlight of the APS April Meeting 2021 is a series of no fewer than four (!) Mini-Symposia on the Muon Collider, held jointly with the DPF. Muon colliders can, in principle, reach the highest lepton collision energies, allowing unprecedented exploration in direct searches of new heavy states and high-precision tests of standard phenomena. However, they still require further studies in various areas, encompassing accelerator, detector and physics. Therefore, to allow reliable predictions of their performance, cost, power consumption and risks, all the community is invited to join discussions at the mini-symposia, and, more generally, to contribute to further developments and technology innovations, in synergy with other more mature concepts.

More information about the APS April Meeting 2021 can be found at https://april.aps.org . 


\section{Book announcements}

This is not a comprehensive list of recent books of interest for the readers of the ICFA Beam Dynamics Newsletter-it is first of all a listing, sorted by publication date, emphasising titles published open access; in addition, a couple of other highly relevant titles have been included.

\subsection{Safety for Particle Accelerators}

Thomas Otto (CERN, Geneva, Switzerland),email: Thomas.Otto@cern.ch.

Publisher: Springer, 30 December 2020, 159 pages, DOI: 10.1007/978-3-030-57031-6.

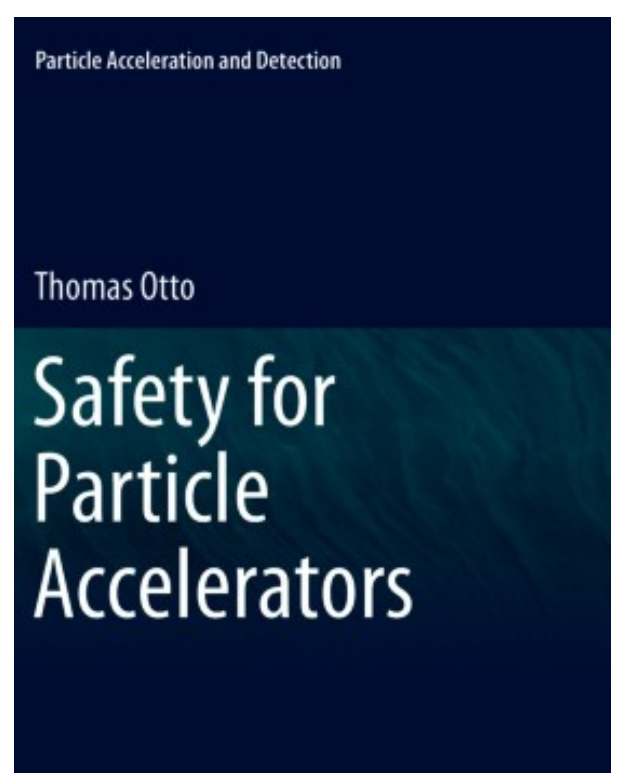

Figure 3. The book is available in open access, hardcovers available from 26 USD.

The use of non-standard technologies such as superconductivity, cryogenics and radiofrequency pose challenges for the safe operation of accelerator facilities that cannot be addressed using only best practice from occupational safety in conventional industry. This book introduces readers to different occupational safety issues at accelerator facilities and is directed to managers, scientists, technical personnel and students working at current or future accelerator facilities. While the focus is on occupational safety - how to protect the people working at these facilities - the book also touches on "machine safety" - how to prevent accelerators from doing structural damage to themselves. This open access book offers a first introduction to safety at accelerator facilities. Presenting an overview of the safety-related aspects of the specific technologies employed in particle accelerators, it highlights the potential hazards at such facilities and current prevention and protection measures. It closes with a review of safety management and organization at accelerator facilities.

\subsection{The Science and Technology of Particle Accelerators}

Robert Appleby (University of Manchester, Manchester, United Kingdom) et al., email:Robert.Appleby@manchester.ac.uk. 
Publisher: CRC Press, 28 December 2020, 322 pages, DOI: 10.1201/9781351007962.

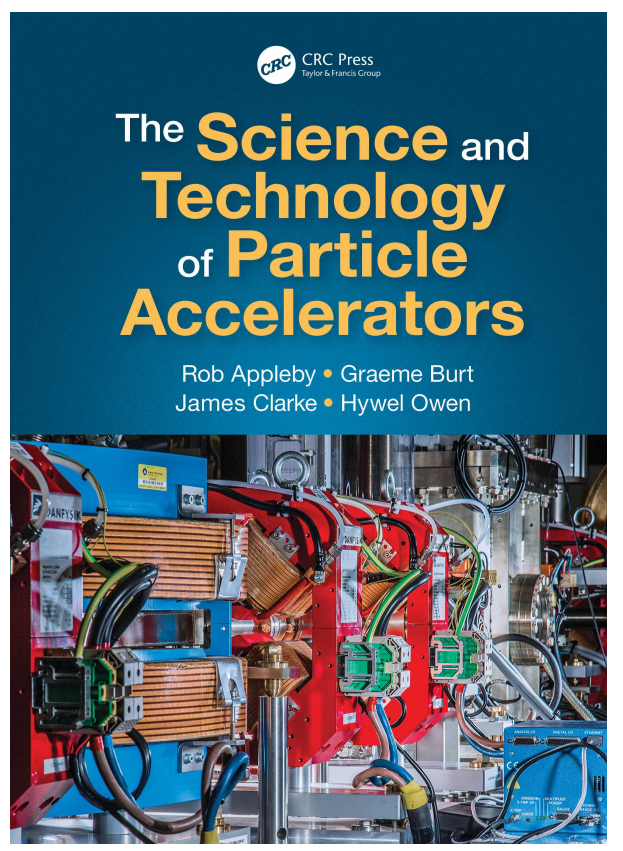

Figure 4. Partial free PDF preview, hardcovers available from 131 USD.

The book provides an accessible introduction to the field and is suitable for advanced undergraduates, graduate students, and academics, as well as professionals in national laboratories and facilities, industry, and medicine who are designing or using particle accelerators. Providing integrated coverage of accelerator science and technology, this text presents the fundamental concepts alongside detailed engineering discussions and extensive practical guidance, including many numerical examples. For each topic, the authors provide a description of the physical principles, a guide to the practical application of those principles, and a discussion of how to design the components that allow the application to be realised.

\subsection{Accelerator Technology: Applications in Science, Medicine, and Industry}

Soeren Moeller (Forschungszentrum Juelich GmbH, Juelich, Germany), email: S.Moeller@fzjuelich.de.

Publisher: Springer, 3 December 2020, 391 pages, DOI: 10.1007/978-3-030-62308-1.

This book explores the physics, technology and applications of particle accelerators. It illustrates the interconnections between applications and basic physical principles, enabling readers to better understand current and upcoming technologies and see beyond the paradigmatic borders of the individual fields. The reader will discover why accelerators are no longer just toys for scientists, but have also become modern and efficient nuclear workhorses. The book starts with an introduction to the relevant technologies and radiation safety aspects of accelerating electrons and ions from several $\mathrm{keV}$ to roughly $250 \mathrm{MeV}$. It subsequently describes the physics behind the interactions of 


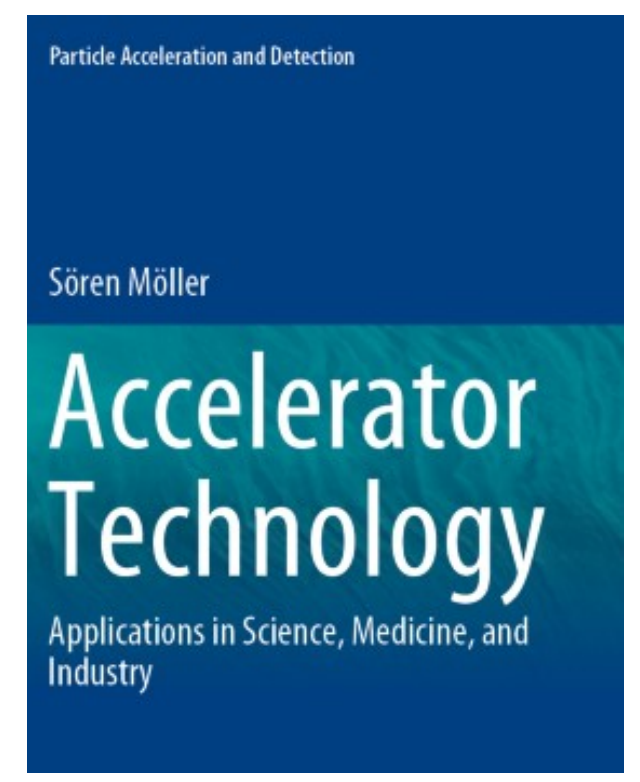

Figure 5. The book is available in open access, hardcovers available from 149 USD.

these particle beams with matter. Mathematical descriptions and state-of-the-art computer models of energy-loss and nuclear interactions between the particle beams and targets round out the physics coverage. On this basis, the book then presents the most important accelerator applications in science, medicine, and industry, explaining and comparing more than 20 major application fields, encompassing semiconductors, cancer treatment, and space exploration. Despite the disparate fields involved, this book demonstrates how the same essential technology and physics connects all of these applications.

\subsection{Particle Physics Reference Library_Volume 3: Accelerators and Colliders}

Eds. Stephen Myers (ADAM SA, Geneva, Switzerland) and Herwig Schopper (CERN, Geneva, Switzerland),email: Stephen.Myers@avo-adam.com.

Publisher: Springer, 28 May 2020, 873 pages, DOI: 10.1007/978-3-030-34245-6.

A joint CERN-Springer initiative, the "Particle Physics Reference Library" provides revised and updated contributions based on previously published material in the well-known Landolt-Boernstein series on particle physics, accelerators and detectors (volumes 21A,B1,B2,C), which took stock of the field approximately one decade ago. Central to this new initiative is publication under full open access. This third volume of the handbook series deals with accelerator physics, design, technology and operations, as well as with beam optics, dynamics and diagnostics. The other two volumes of the full set are v.1 : Theory and experiments and v.2 : Detectors for particles and radiation. A full review of the work will appear in the CERN Courier later this spring. 


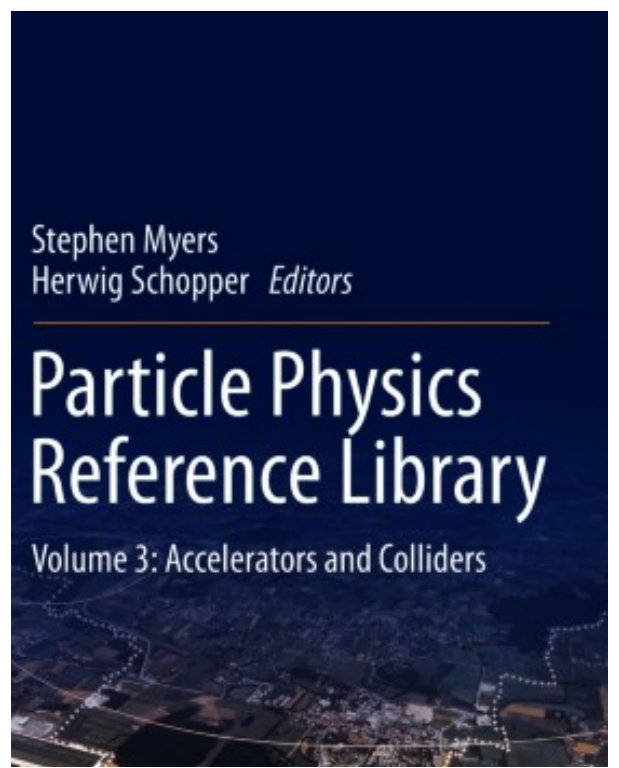

Figure 6. The book is available in open access, hardcovers available from 55 USD.

\subsection{Obsessed by a Dream: The Physicist Rolf Wideroe-a Giant in the History of Accelera- tors}

Aashild Sorheim (Oslo, Norway), email: aashildsorheim@mac.com.

Publisher: Springer, 7 November 2019, 545 pages, DOI: 10.1007/978-3-030-26338-6.

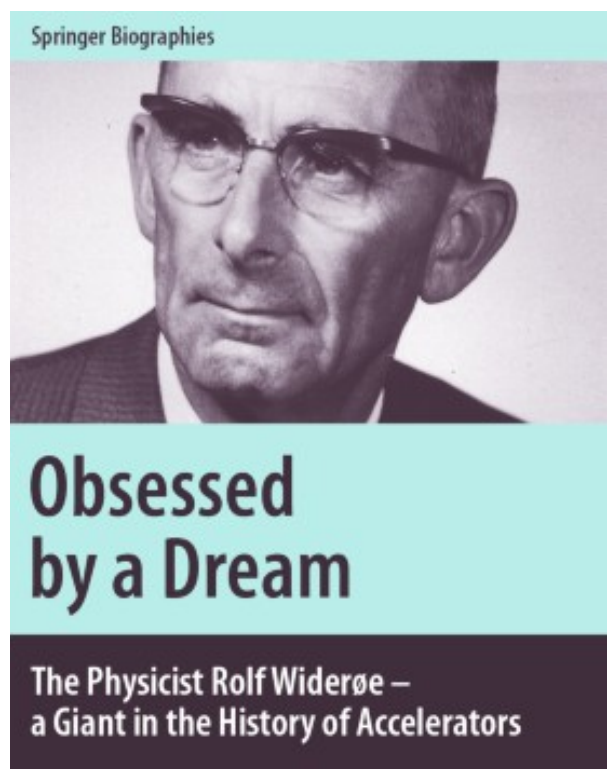

Figure 7. The book is available in open access, hardcovers available from 49 USD.

The first of Wideroe's many pioneering contributions in the field of accelerator physics was the betatron. He later went on to build the first radiation therapy machine, an advance that would 
eventually revolutionize cancer treatment. Hospitals worldwide installed his machine, and today's modern radiation treatment equipment is based on his inventions. Wideroe's story also includes a fair share of drama, particularly during World War II when both Germans and the Allies vied for his collaboration. Wideroe held leading positions in multinational industry groups and was one of the consultants for building the world's largest nuclear laboratory, CERN, in Switzerland. He gained over 200 patents, received several honorary doctorates and a number of international awards. 